\title{
Constrictive Pericarditis in a 19-Month-Old Child
}

\author{
José Carlos dos Santos G uitti
}

\author{
Londrina, PR - Brazil
}

\begin{abstract}
We report a case of chronic nonspecific constrictive pericarditis with myocardial involvement in a 19-monthold infant. The patient underwent total pericardiectomy and had irreversible hemodynamic instability. Constrictive pericarditis is rare in childhood. It may follow several processes, most frequently an infectious disease. The natural course of the disease consists of progressive myocardial dysfunction with atrioventricular valvular involvement. When diagnosis is established early in the course of the disease and treatment started immediately, the evolution is favorable in most instances.
\end{abstract}

Constrictive pericarditis in children is, in the majority of cases, a diagnostic challenge because it is rare and therefore not readily recalled, has an uncharacteristic clinical picture, and is capable of mimicking other diseases.

Constrictive pericarditis can develop from infectious processes, collagenosis, uremia, neoplasias, radiation, traumatisms and granulomatous diseases ${ }^{1,2}$. It has been described as a complicating factor in cardiac surgery and myocardial infarction ${ }^{3}$, as well as a feature of the mulibrey nanism syndrome ${ }^{4}$. In spite of numerous known causes, constrictive pericarditis often occurs in the absence of evident previous illness ${ }^{1}$. Even when the process does have an infectious etiology, it is not possible in approximately $60 \%$ of cases to identify the responsible agent ${ }^{5}$.

Two forms of the disease are recognized: a chronic one, which is usually not possible to relate to an etiological agent, and another, of rapid onset, subsequent to acute pericarditis with fluid accumulation ${ }^{6}$.

Constrictive pericarditis is not common in children; of 235 pericarditis patients treated at the Toronto Hospital for Sick Children over approximately 30 years, only two developed pericardial thickening and constriction ${ }^{7}$. Detection in infants is rather rare. Grumach et al. ${ }^{3}$ described it as a complication in a 17-month-old patient with chronic granulatomatous disease. In Vining's review ${ }^{8}$, the youngest patient was two years old. It is possible that such a low incidence is due, in part, to lack of awareness of its possible occurrence.

Universidade Estadual de Londrina.

Mailing address: José Carlos dos Santos Guitti - Rua Paranaguá, 934 - 86020-030 - Londrina, PR - Brazil

Received on $4 / 1 / 99$

Accepted on 5/12/99
Furthermore, diagnosis of acute pericardial fluid accumulation without occlusion can pass unnoticed especially when of viral origin, not leading therefore to an adequate follow-up of the patient. Even cases subsequent to tuberculosis may not be correctly diagnosed, because of the insidious character of this disease and its complications.

At the pediatric clinic of the University Hospital of Londrina in the period between 1989 and 1998, only five cases of acute nonrheumatoid pericarditis showed sufficiently clear-cut clinical manifestations to be diagnosed as constrictive pericarditis. The disease presented in all patients as a complication of bacterial pulmonary infection or sepsis, with the exception of the one presently described, whose cause could not be determined in spite of intensive laboratory investigation.

\section{Case Report}

The patient was a white 19-month-old female. When 14 months old, she had pericardial effusion during infection of the upper air airways, presumed to be of viral origin in view of the lack of specific laboratory results. She remained well for four months after which she started to show progressive respiratory discomfort, accompanied by asthenia, irritability and a painful abdomen. A clinical examination revealed that she had discrete tachypnea, nonpulsatile hepatomegaly, jugular stasis and tachycardia. The second heart sound had a variable splitting and hyperphonesis of the pulmonary component was observed. Pericardial clicking or attrition could not be detected. Mild Rhonchi and rattling were noted in both lungs. No evidence of edema or ascites existed. A chest film showed a small enlargement of the cardiac area at the expense of the right chambers, as well as signs of pulmonary circulatory stasis with intercisural fluid accumulation. Electrocardiogram showed only decreased amplitude of QRS complexes, nonspecific alterations of ventricular repolarization and signs of biatrial hypertrophy. An echocardiogram detected a small accumulation of pericardial fluid with thickening of the parietal leaflet, without calcification; increased atrial and right ventricular chambers, with dilated inferior cava and suprahepatic veins, with reversed flow; pulmonary artery hypertension $(45 \mathrm{~mm}$ $\mathrm{Hg}$ ), tricuspid regurgitation and atrioventricular valve flow with a protodiastolic type of filling (figures 1,2 and 3). The diagnosis of constrictive pericarditis was confirmed by hemodynamic evaluation. The patient's family opposed 
surgical intervention, and the patient returned to the clinic after eight months, presenting with signs of cardiac heart failure and marked malnutrition. Due to the poor clinical condition of the patient, only partial pericardiectomy was performed, in an attempt to obtain some degree of decompression. Discrete improvement of symptoms occurred. After another long period of seven months without medical follow-up, she was readmitted to the hospital presenting with clinical signs of serious deterioration in myocardial function. Following partial recovery of her nutritional status, she underwent a radical pericardiectomy, tricuspid valvoplasty and closure of the interatrial communication of the ostium secundum type (surgical finding). She had renal failure and hemodynamic instability, and died on the $26^{\text {th }}$ day. The autopsy documented chronic nonspecific pericarditis.

\section{Discussion}

Clinical manifestations of constrictive pericarditis result mainly in decreased ventricular filling and impaired myocardial contractility, although systolic function can also be altered to a varying degree. These alterations lead to the progressive depression of cardiac function.

Experimental reproduction of constrictive pericarditis by fixation of a rigid plastic cover over the heart is only successful when ventricular compression is achieved. When restricted to the atrial cavities, it does not lead to the same phenomenon. Thus the disease acts in a way similar to mitral and tricuspid stenosis. As a result, high initial filling pressures in both ventricles, small ejection volume and low cardiac output are observed ${ }^{5}$.

The symptoms have a slow and insidious progress, but in some cases intense, early myocardial damage can accelerate the course of the disease. The clinical condition is in general restricted to systemic and pulmonary congestion, not accompanied by a degree of dyspnea proportional to that of stasis. In about $75 \%$ of cases, Beck's triad is found, consisting of little symptomatic evidence of cardiac dysfunction, increased venous pressure and ascites, not

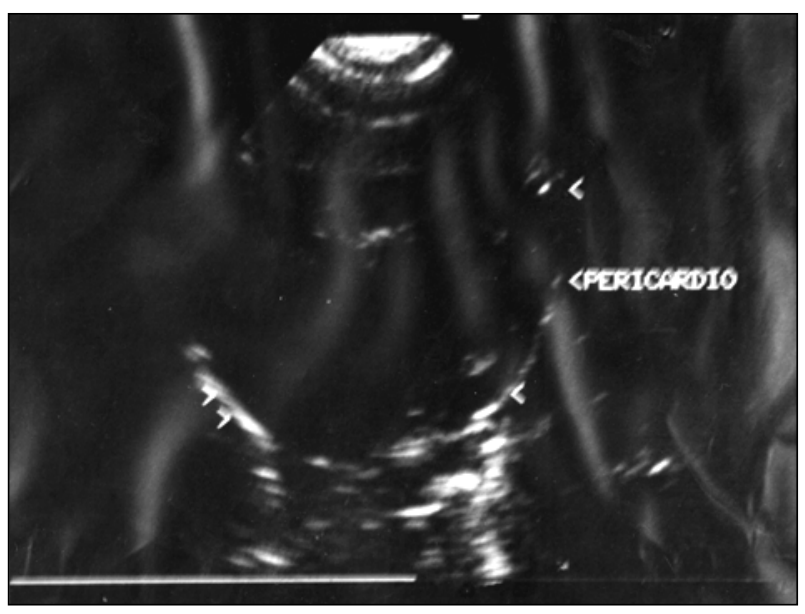

Fig. 1- Echocardiography. Pericardial thickening ( arrows).

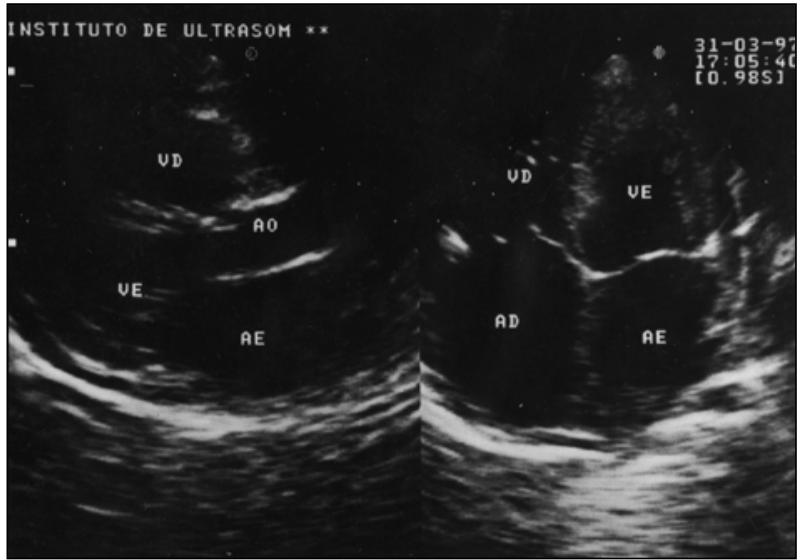

Fig. 2 - Echocardiography. Mild fluid accumulation and pericardial thickening (left). Dilatation of the atrial cavities (right).

proportional to the usually discrete or nonapparent edema ${ }^{9}$. Hepatomegaly is intense and non-pulsatile. This fact, coupled with the possible presence of hypoproteinemia, mimics chronic hepatic disease and sometimes primary malnutrition. The occurrence of ascitis, edema and hypoproteinemia raises, in some cases, the diagnostic hypothesis of nephrotic syndrome. Cardiomegaly associated with systolic murmurs in mitral and tricuspid areas and to further signs of restriction may lead to the suspicion of the existence of restrictive cardiomyopathy ${ }^{1}$.

Hypoproteinemia occurs as a manifestation of proteinlosing enteropathy and may be accompanied by steatorrhea and significant signs of malnutrition. Under these circumstances, the first hypothesis raised is a malabsorption syndrome.

The mulibrey nanism syndrome (muscle, liver, brain, eyes), in addition to pericardial constriction and low stature, is characterized by yellow retineal pigmentation, fibrous bone displasia and a J-shaped sela turcica ${ }^{4}$.

The multiplicity of differential diagnosties explains why, on some occasions, clinical investigation takes a long time, allowing for the unrestricted evolution of the disease due to the delay in obtaining confirming evidence.

The chest film, a usual complementary exam, reveals in most cases the, pulmonary venous stasis and normal or slight increase in the cardiac silhouette. A small pleural effusion may be present. Pericardial calcification is detected in approximately $50 \%$ of cases ${ }^{5,10}$.

Electrocardiographic characteristics are restricted to low voltage of QRS complexes and nonspecific alterations in ventricular repolarization. The altered ST segment, a characteristic sign of acute pericarditis, is not observed. Alterations in the $\mathrm{P}$ wave and arrhythmias, especially atrial fibrillation, are only present when myocardial damage is extensive ${ }^{10}$.

Echocardiography does not allow an accurate evaluation of the degree of constriction. Depending on the degree of pericardial thickening, echoes of corresponding density are obtained. The ventricular septum has paradoxical movement, and the movement of the posterior free wall of 


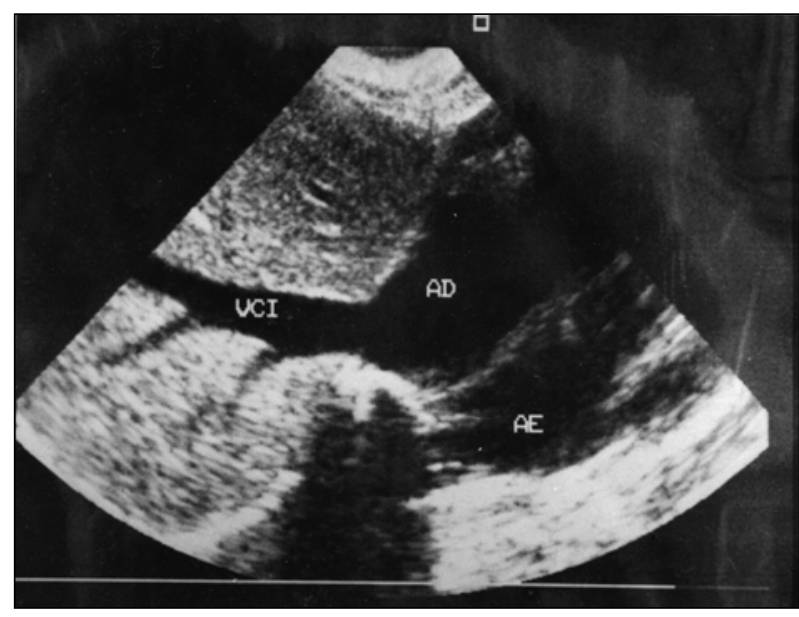

Fig. 3 - Echocardiography. Pronounced dilatation of the inferior vena cava.

the left ventricle produces sudden angulation of echoes between the protodiastolic phase and the remaining diastole ${ }^{10}$.

The most characteristic alteration obtained by cardiac catheterization can also be detected by echocardiography. It consists of rapid initial ventricular filling, abruptly interrupted by the limitation of ventricular distensibility, which produces increased down sloping in the diastolic segment of the mitral valve, with $\mathrm{E}>>\mathrm{A}^{10}$.

Occasionally, despite all available investigational means, a definitive diagnosis can only be obtained by exploratory thoracotomy ${ }^{1}$.

As in cases of cardiac outflow obstruction, the use of digitalis, diuretics and vasodilators is contraindicated, because tachycardia, increased venous pressure and peripheral vasoconstriction are compensatory hemodynamic responses ${ }^{11}$. The only treatment to be considered is surgery, to be performed as soon as possible. Partial pericardiotomy may be indicated, as was pointed out by Nataf et al. ${ }^{12}$, who resorted to this procedure in 75 of 84 patients, 7 of which required total pericardiotomy at a later date. The majority of authors recommend radical pericardiectomy, including the region of origin of the great arteries. In most cases a rapid response occurs characterized by increased cardiac output, abundant diuresis and regression of the signs of venous congestion. As pointed out by Gersony and Allan ${ }^{1}$, the dramatic recovery of the general conditions

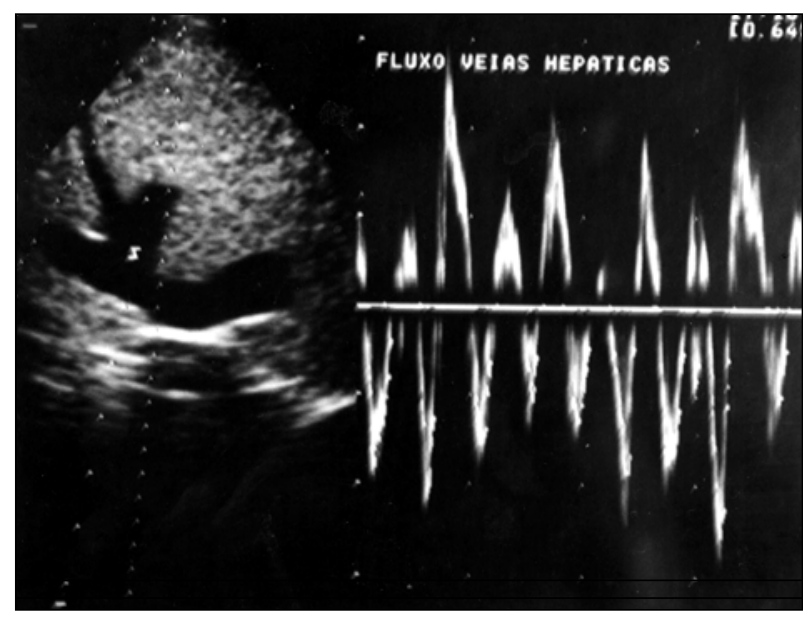

Fig. 4 - Echocardiography. Dilatation and bidirectional flow in supra-hepatic veins.

of a child with hitherto unsuspected chronic constrictive pericarditis is a highly rewarding experience in medicine. However, when myocardial damage has been severe as observed in the present case, ventricular dysfunction and failure to decompression may occur immediately in the postoperative period, leading to hemodynamic instability that is difficult to reverse ${ }^{5}$.

Tricuspid or mitral valvuloplasty is indicated in cases of significant regurgitation, because its spontaneous regression is a long process and only occurs in $50 \%$ of patients ${ }^{13}$.

The present report illustrates the need for early surgical intervention, even during the initial stage of the disease. Unfortunately, as well pointed out by Allen et al. ${ }^{14}$, the sociocultural condition of the less protected strata of our population often results in difficulty to provide the most adequate medical and hospital care.

In cases of tuberculosis or hystoplasmosis, the use of specific therapeutic agents during the pre-operative phase is recommended, and such treatment should continue until full resolution of the infection has been achieved.

\section{Acknowledgments}

The Author thanks Dr. Paulo Sampaio Gutierrez and Dr. Maria Irma Seixas Duarte for the pathological and immunohistochemical exams, respectively.

\section{References}

1. Gersony WM, Allan MA. Infective endocarditis and diseases of the pericardium. Pediatr Clin North Am 1978; 25: 833-45.

2. Grumach AS, Jacob CMA, Stolf NG, Maksoud JC, Carneiro-Sampaio MS Pericardite constritiva como complicação de doença granulomatosa crônica na infância. Rev Hosp Clin FMUSP 1987; 42: 30-2.

3. Cohen MV, Greenberg MA. Constrictive pericarditis: early and late complication of cardiac surgery. Am J Cardiol 1979; 43: 657-61.

4. Voorhess ML, Husson GS, Blackman MS. Growth failure with pericardial constriction. The syndrome of mulibrey nanism. Am J Dis Child 1976; 130: 1146-8.
5. Cayler GG, Riley HD, Mc Henry MM. Constrictive pericarditis. In: Moss AJ, Adam FH. Heart Disease in Infants, Children and Adolescents. Baltimore: The Williams \& Wilkins Co., 1968: 859.

6. Strauss AW, Santa-Maria M, Goldring D. Constrictive pericarditis in children Am J Dis Child 1975; 129: 822-6.

7. Keith JD. Constrictive pericarditis. In: Rowe RD, Vlad P. Heart Disease in Infancy and Childhood. $2^{\text {nd }}$ ed. New York: The McMillan Co., 1978: 255-8.

8. Vining CW. Constrictive pericarditis in early childhood. Proc Roy Soc Med 1955; 48: 1103.

9. Beck CS. Two cardiac compression triads. JAMA 1935; 104: 714 
10. Treviño CP. Enfermedades del pericardio. In: Sanchez PA. Cardiologia Pediatrica. Clinica y Cirugia. Barcelona: Salvat Ed. SA, 1986: 930.

11. Nadas AS. Pediatric Cardiology. $2^{\text {nd }}$ ed. Philadelphia: Saunders Company, 1963: 300

12. Nataf $P$, Cacoub $P$, Dorent R, et al. Results of subtotal pericardiectomy for constrictive pericarditis. Eur J Cardio Thorac Surg 1993; 7: 252-5.
13. Mantri RR, Radhakrisnan S, Sinha N, Goel PK, Bajaj R, Bidwai PS. Atrioventricular regurgitations in constrictive pericarditis: incidence and postoperative outcome. Int J Cardiol 1993; 38: 273-9.

14. Allen HD, Taubert KA, Deckelbaum RJ, et al. Poverty and cardiac disease in children. Am J Dis Child 1991; 145: 550-3. 